\title{
LAS WEBQUEST Y EL MODELO CAIT, COMO MODELOS DE ENSEÑANZA APRENDIZAJE EN LA RED INTERNET
}

\author{
(THE WEBQUEST AND MODEL CAIT, EDUCATION MODELS LEARNING IN NETWORK
}

\section{INTERNET)}

José Tortajada

Catedrático numerario de Bachillerato (España)

\section{RESUMEN}

Se muestra como se puede enseñar y aprender utilizando Internet, a través de una metodología contrastada, el modelo CAIT y con una herramienta muy versátil y fácil de utilizar las Webquest. Se proporcionan varios ejemplos, uno en el artículo y otros en la página web del profesor. Y, se explica como se puede llevar a cabo la enseñanza con este método y como podemos adaptarnos a la misma.

\begin{abstract}
In this publication, one is as it is possible to be taught and to be learned using Internet, through a resisted methodology, model CAIT and with a tool very versatile and easy to use the Webquest. Several examples, one in the article and others in the page are provided Web of the professor. And, it is explained as education with this method can be carried out and as we can adapt to the same one.
\end{abstract}

\section{WEBQUEST SOBRE:}

ASTRONOMÍA: LA LUNA, MARTE, VENUS, COORDENADAS TERRESTRES Y CELESTES, LAS GALAXIAS, EL SISTEMA SOLAR.

GEOLOGÍA Y BIOLOGÍA: EL ORIGEN DE LA VIDA, EL ORIGEN DE LOS ESPAÑOLES, LAS ROCAS, ORIGEN DE LAS ROCAS SEDIMENTARIAS, LA CONTAMINACIÓN DE LOS RÍOS, DEL OZONO, EL AGUA, LA ENERGÍA SOLAR. 
JOSÉ TORTAJADA

LAS WEBQUEST Y EL MODELO CAIT COMO MODELOS DE ENSEÑANZA APRENDIZAJE EN LA RED INTERNET

\section{INTRODUCCIÓN}

\section{¿QUÉ ES UNA WEB QUEST?}

WebQuest se define como una actividad de búsqueda informativa guiada, en la cual la mayor parte de la información empleada por los alumnos es extraída de la red.

La creación del término WQ y el desarrollo de la idea del mismo modelo, como método de aprendizaje se deben al profesor de la Universidad de San Diego Bernie Dodge, que elaboró una teoría de este nuevo sistema en 1995.

Para profundizar sobre este modelo y acceder al artículo en que este autor, da forma al modelo WQ, pueden visitar las siguientes páginas:

\section{http://edweb.sdsu.edu/courses/edtec596/about webquests.html}

http://www.ozline.com/webquests/intro.html

Las Webquest, son páginas webs preparadas con objetivos formativos o educativos y organizadas de determinada manera para conseguir el aprendizaje de sus interrogantes, sus contenidos, o para conseguir unos objetivos formativos, entre otras acciones educativas (Rosado L., y Tortajada, J., 2002, a, b y 2003).

Por ello contienen diversas actividades de enseñanza / aprendizaje, creadas para que al utilizar Internet en la educación, consigamos formar, educar, enseñar y aprender, e integrarlo en el proceso de Enseñanza-Aprendizaje, a su vez son el resultado de la utilización de un método de enseñanza basado en las estrategias de aprendizaje por descubrimiento, guiándose con ellas un proceso de trabajo desarrollado por los estudiantes o alumnos, utilizando los recursos de la WWW.

Cuando Bernie Dodge en 1995 creó este modelo lo definió como "una actividad orientada a la investigación donde toda o casi toda la información que se utiliza procede de recursos de las Webs".

"A WebQuest is an inquiry-oriented activity in which some or all of the information that learners interact with comes from resources on the internet" 
JOSÉ TORTAJADA

LAS WEBQUEST Y EL MODELO CAIT COMO MODELOS DE ENSEÑANZA APRENDIZAJE EN LA RED INTERNET

Con las Webquest, es posible integrar los principios del aprendizaje constructivista, la metodología de enseñanza por proyectos y la navegación web para desarrollar el curriculum con grupos de alumnos de un aula ordinaria.

Una Webquest, además consiste en presentarle al alumnado un problema o un conjunto de interrogantes, con una guía del proceso de trabajo que deben llevar a cabo y un conjunto de recursos preestablecidos y accesibles a través de la WWW.

Esta actividad se aborda en la forma de un trabajo, que se puede realizar en un grupo en el aula, en un pequeño grupo, aunque también se puede preparar para realizarlo individualmente en el aula o en casa y con el los alumnos deben elaborar un trabajo (bien en papel o en formato digital) con los resultados del mismo.

Este tipo de trabajo, puede adaptarse a las situaciones en las que no todos los alumnos tienen el ordenador delante, e incluso parte del mismo se puede hacer off - line o puede llevarse a cabo en las aulas en las que hay uno o varios ordenadores aislados del resto del grupo para que trabajen en el aula en pequeño grupo de investigación.

Como indica J. Adell (2002) una WebQuest es una actividad didáctica atractiva para los estudiantes, y que les permite desarrollar un proceso de pensamiento de alto nivel. "Se trata de hacer algo con la información: analizar, sintetizar, comprender, transformar, crear, juzgar, valorar, etc."

Una WebQuest (B. Dodge y T. March), se compone de seis partes esenciales: Introducción, Tarea, Proceso, Recursos, Evaluación y Conclusión.

\section{¿QUÉ Y CÓMO ESTUDIAR Y APRENDER?}

Al plantearnos utilizar esta potente herramienta, Internet, en el proceso de enseñanza aprendizaje, al tratar de sacarle partido a este inmenso avance que significa poner en manos de la sociedad, y de los estudiantes en particular, toda (o casi toda) la información existente por escrito o en papel (digital o electrónico) en la humanidad, nos hemos de nuevo plantear los profesores lo que queremos enseñar y lo que hay que hacer para aprender, para formar, para educar, etc., en lo que es nuestro trabajo el de preparar a los niños y jóvenes (o adultos) para su integración laboral, familiar, social, para su desarrollo integral como personas, para que aprendan a pensar, a ser analíticos, críticos, independientes y socializados, para que desarrollen la inteligencia emocional, la intelectual, la memoria, etc., etc. 
JOSÉ TORTAJADA

LAS WEBQUEST Y EL MODELO CAIT COMO MODELOS DE ENSEÑANZA APRENDIZAJE EN LA RED INTERNET

¿Qué nos proponemos a la hora de enseñar, estudiar y aprender y cómo lo hacemos?

\section{USANDO INTERNET COMO MÉTODO DE ENSEÑANZA CON LAS "WEB QUEST"}

\section{La interpretación del significado de lo que son las WEB QUEST es: BÚSQUEDAS GUIADAS en Internet.}

Las WebQuest son actividades de enseñanza - aprendizaje enfocadas a la investigación en las que los estudiantes (individualmente o en grupo) realizarán una serie de tareas que exigirán procesos de análisis, evaluación, síntesis, argumentación... a partir de la consulta de diversas fuentes de información sugeridas (la mayoría de ellas páginas web de Internet).

Carmen Barba, especialista en WebQuest y autora de diversas páginas web sobre el tema, matiza (traduciéndolo nosotros): "La manera de trabajar con las WQ está estructurada de tal manera que garanticen que el trabajo en grupo consigue de manera imprescindible y comprometida a todos y cada uno de los miembros del grupo, al deber demostrar y compartir con los otros las investigaciones y trabajos individuales, a la vez que cada uno es responsable de una de las partes del trabajo que han de poner en común.

Su duración puede ser variable, tanto en número de sesiones como en su distribución a lo largo del tiempo. En algunos casos simplemente se pretenderá que los estudiantes entren en contacto con determinadas informaciones generales relacionadas con una temática, y las estructuren a partir de unos criterios o perspectivas (Webquest cortas QuickQuest las más cortas). En otros casos se pretenderá que los estudiantes amplíen o profundicen en algún aspecto concreto de sus conocimientos, elaborando algún nuevo material o realizando nuevas aplicaciones de estos contenidos (Webquest largas).

Los temas de actualidad y los hechos que pueden suscitar diversos posicionamientos o explicaciones resultan especialmente adecuados para estas actividades, que sitúan a los estudiantes ante una investigación en la que deben implicarse a partir de sus puntos de vista. 


\section{EL MODELO CAIT}

Un modelo bastante nuevo y compatible con el uso de las Webquest es el modelo: CAIT denominado también como de Estrategias para una nueva pedagogía.

Beltrán y Pérez (2003, a) crean con su equipo un modelo de enseñanza con Internet que sistematiza un modelo de aprendizaje constructivista, autorregulado, interactivo y tecnológico (modelo CAIT).

En sus planteamientos de lo que es la enseñanza y el aprendizaje, Beltrán expone el siguiente esquema del aprendizaje:

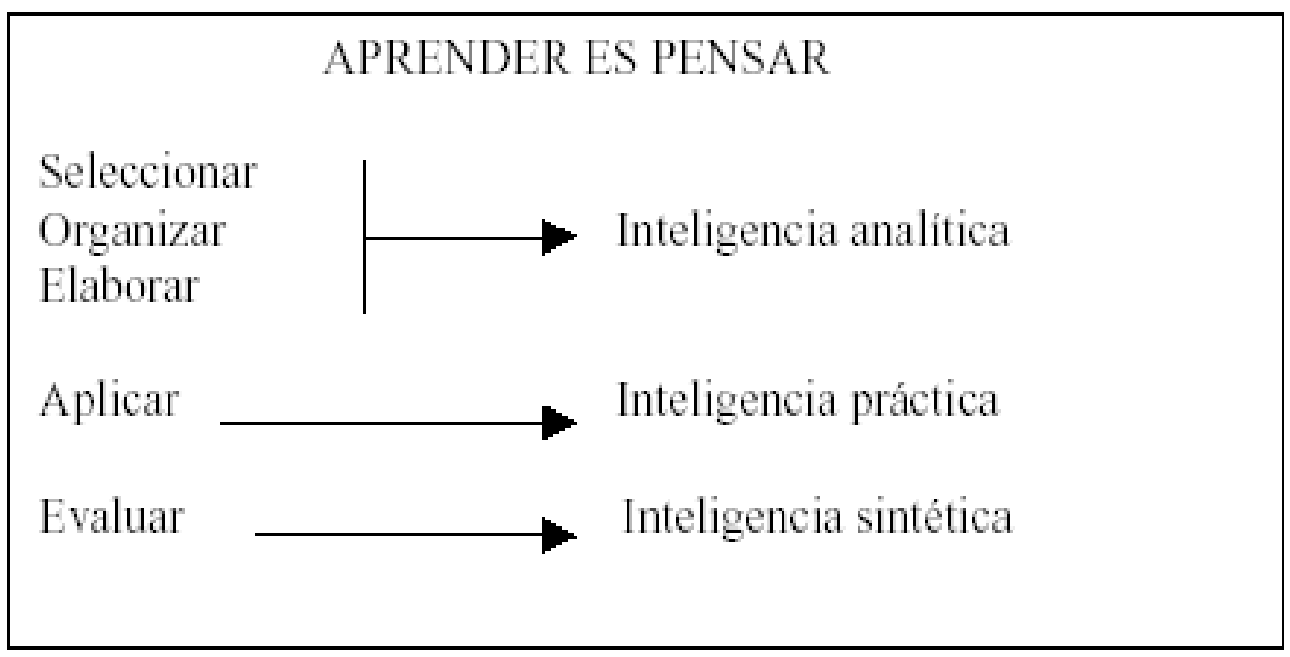

La clasificación de los instrumentos que podemos utilizar para el aprendizaje se basa en las estrategias cognitivas que cada instrumento desarrollan de una manera directa, aunque un mismo instrumento puede promover varias estrategias cognitivas en diferentes grados. Siguiendo a Jonassen (2000) una buena clasificación sería esta: 


\begin{tabular}{|c|c|}
\hline \multicolumn{2}{|c|}{ INSTRUMENTOS COGNITIVOS } \\
\hline \multicolumn{2}{|c|}{$\begin{array}{l}\text { 2.ORGANIZAR LA INFORMACIÓN: BASES DE DATOS, ANÁLISIS DE DOMINIOS E } \\
\text { HISTORIAS }\end{array}$} \\
\hline $\begin{array}{l}\text { 3.ELABORAR LA INFORMACIÓN: } \\
\text { TABLAS, DIAGRAMAS }\end{array}$ & CONCEPTUALES, \\
\hline $\begin{array}{l}\text { 4.REPRESENTAR CL CONOCIMIENTO: } \\
\text { SIMULACIONES, HIPERMEDIA }\end{array}$ & MICROMUNDOS, \\
\hline 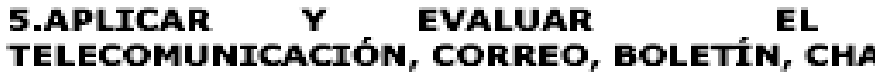 & $\begin{array}{l}\text { CONOCIMIENTO: } \\
\text { S }\end{array}$ \\
\hline
\end{tabular}

La investigación educativa no ha encontrado nunca un modelo didáctico que sea mejor que todos los demás. Cada modelo tiene sus ventajas y desventajas. Los modelos que mejor se ajustan al nuevo paradigma educativo centrado en el alumno $\mathrm{y}$ en su aprendizaje son los que figuran en el cuadro adjunto que representa las seis arquitecturas modélicas:

\begin{tabular}{|l|}
\hline \multicolumn{1}{|c|}{ LAS SEIS ARQUITECTURAS } \\
•MÉTODO DE SOLUCIÓN DE PROBLEMAS \\
•MÉTODO DE PROYECTOS \\
•MÉTODO DE CASOS \\
•MÉTODO SOCRÁTICO \\
•MÉTODO ESTRATÉGICO \\
•MÉTODO C.A.I.T. \\
\hline
\end{tabular}

El alumno en el mismo necesita discriminar la información relevante, de la no relevante y con ella debe elaborar su síntesis. Para realizar con éxito este proceso debe seleccionar, organizar y elaborar la información y por lo tanto debe aprender a realizar todas estas acciones. 
La evolución de este modelo, hasta el presente se está produciendo dentro del contexto de la lengua castellana, gracias a Internet y a EDUCARED, que tiene importantes extensiones en el área iberoamericana, en contraste con otros modelos de enseñanza anglosajones que como el modelo Webquest resultan compatibles con el modelo CAIT e incluso se enriquecen mutuamente.

En el segundo Congreso Educared (2003), y con respecto al modelo CAIT, Soler Areta (2003), decía:

Utilizando el modelo CAIT, con los alumnos, en la primera sesión lo que hacemos es una sesión de presentación. Se plantea a los alumnos el título genérico de la unidad que se va a trabajar; se pide que recuerden qué saben sobre ello, se les plantea los objetivos que se pretenden, formulados en forma de tareas, asimismo se les plantea las herramientas que se van a utilizar y se les da las normas de todo el proceso.

Durante las siguientes sesiones se plantea a los alumnos un cuestionario de trabajo. Ese cuestionario está disponible en el navegador Educared en la página del profesor. Ahí mismo también se señalan los recursos a los que el alumno puede acudir para hacer su síntesis.

Para lograr esa síntesis el profesor, además le facilita ejercicios de aplicación (posteriores a la síntesis) realizados con Clic, o los que el libro de texto señala en la página que el alumno, a su elección, debe realizar el trabajo sobre el texto.

Cuando el alumno juzga que está preparado, realiza un examen que tiene a su disposición en su dirección de correo electrónico o en la página web. Es un examen, que si se realiza utilizando la herramienta "TestIt" (que se encuentra entre los recursos didácticos del Educared -www.educared.net) que se corrige automáticamente y muestra en el momento los resultados, la nota y los fallos y envía el resultado al profesor, además de mostrárselo al estudiante.

En una fecha previamente acordada, el profesor (o quien el profesor decida) realiza una síntesis de las síntesis realizadas por los alumnos, recogiendo y haciendo hincapié en aquellos aspectos, a su juicio esenciales y, completando o matizando algún extremo que crea necesario.

La evaluación de la unidad puede realizarse casi individualmente, dialogando con los alumnos los resultados y en ella se tiene en cuenta la síntesis personal del tema, las notas de las evaluaciones automáticas y la nota del examen final. 
JOSÉ TORTAJADA

LAS WEBQUEST Y EL MODELO CAIT COMO MODELOS DE ENSEÑANZA APRENDIZAJE EN LA RED INTERNET

\section{HACER UNA WEBQUEST ADAPTÁNDOLA AL MODELO CAIT.}

Cada Webquest se concreta en un documento que tiene los siguientes apartados:

1. Título de la Webquest

2. Introducción.

3. Resumen de la actividad.

4. Presentación de la situación o escenario (debe resultar atractivo, a veces los alumnos reciben una misión y adoptan un rol: detective, periodista...)

5. Actividad. Descripción de las tareas a realizar (que pueden estar relacionadas con una asignatura determinada o ser interdisciplinarias) y de los objetivos formativos que se pretenden. Las actividades pueden centrarse en la resolución de un problema, la elaboración de una síntesis, contestar determinadas preguntas... En cualquier caso, deben resultar interesantes para sus destinatarios.

6. Recursos. Listado de materiales que se utilizarán: páginas web, foros telemáticos y otros libros y documentos que sean de fácil acceso para los destinatarios de la actividad. Conviene incluir una breve presentación de cada recurso indicando la información que proporcionan.

7. Proceso. Descripción del proceso a seguir para realizar las tareas, con indicación de cada uno de los pasos que los estudiantes deben realizar.

8. Evaluación. Los resultados obtenidos por los estudiantes se presentan y discuten públicamente en clase. También pueden publicarse en Internet (en su web personal, en la web del centro...); de esta manera el trabajo puede ser compartido y comentado por una mayor audiencia, y la motivación de los alumnos puede ser mayor.

9. Consejos y sugerencias. Se pueden incluir orientaciones diversas: estructuración de la información (esquemas, mapas cognitivos...), análisis (aspectos a considerar), valoración (posibles criterios)...

10. Conclusión. Comentarios finales sobre la actividad y pautas para la presentación de los resultados obtenidos: organización de la información, aspectos clave.

Y, ¿̇cómo adaptarla al modelo CAIT? 
JOSÉ TORTAJADA

LAS WEBQUEST Y EL MODELO CAIT COMO MODELOS DE ENSEÑANZA APRENDIZAJE EN LA RED INTERNET

Recordemos que: CAIT integra los conceptos constructivo, autorregulado, interactivo y tecnológico.

Nuestra forma de llevarlo a cabo es la siguiente:

1. En primer lugar el profesor contextualiza el tema, define y expone los objetivos, los contenidos mínimos, introduce o/y explica el tema y expone el papel del alumno y su grado de libertad.

2. Los alumnos investigan en Internet, ven las presentaciones y los vídeos.

3. Realizan las actividades con Internet, con los programas de ordenador, en papel o en laboratorio y completan sus apuntes, el cuaderno de actividades y las conclusiones

4. Se realiza la evaluación de su aprendizaje con el "cuaderno de trabajo, el dossier de actividades y las pruebas de conocimientos y aprendizajes”.

\section{EJEMPLOS:}

Los cursos en los que lo llevamos a cabo, son todos los que tenemos los profesores participantes, es decir: Ciencias de la Tierra y del Medio ambiente de $2^{\circ}$ de Bachillerato, Taller de $4^{\circ}$ de ESO Astronomía de $4^{\circ}$ de ESO, Medio Ambiente y Energías renovables de $3^{\circ}$ ESO y Biología y Geología de $3^{\circ}$ de ESO.

En cuanto al tiempo que les dedicamos con ellos, es del orden de dos a tres semanas por tema mencionado.

El aula que utilizamos es la de ordenadores en que tenemos todo el horario de los alumnos con el aula disponible.

¿Porqué Introducir estas unidades de esta forma?

Creemos que los profesores además de enseñarles una materia, hay que enseñarles a aprender, a construir su conocimiento, a adquirir aprendizajes de destrezas y habilidades propias de las Ciencias, hay que educarles en valores y hay que formarles para que puedan seguir aprendiendo por sí solos, en lo que necesiten y que lo sepan hacer utilizando Internet.

Además de que Internet y los ordenadores son una herramienta motivadora, son parte del aprendizaje que deben de aprender a aprender para este futuro tecnológico. 
JOSÉ TORTAJADA

LAS WEBQUEST Y EL MODELO CAIT COMO MODELOS DE ENSEÑANZA APRENDIZAJE EN LA RED INTERNET

\section{LAS GALAXIAS}

Objetivos: conocer la principal estructura del Universo, las Galaxias, su evolución, como nacieron, y de qué están hechas, es lo que haremos a través de este sistema de enseñanza que utiliza un modelo de enseñar a aprender con los Ordenadores e Internet y que se denomina modelo CAIT.

Metodología: esta vez no será el profesor quien te explique el tema, te lo introducirá, te proporcionará las pautas a seguir en su estudio, te ayudará en los trabajos que debes hacer y finalmente te evaluará.

Vamos a utilizar las Tecnologías de la Información, a través de los ordenadores, utilizarás Internet e Intranet (red interior del centro) e igualmente verás documentales a través de los ordenadores y realizaremos algunas prácticas de reconocimiento de rocas.

\section{PASOS}

1.- En primer lugar se te pasará una prueba de test de conocimientos previos, que harás bien en papel o en formato electrónico tal y como te explique el profesor.

En varios de los pasos siguientes, el profesor te ofrecerá o pedirá que veas una serie de vídeos sobre las Galaxias, que podrás visualizar personalmente en tu ordenador.

2.- El segundo paso consistirá en que tu mismo verás la presentación de power point, en tu ordenador, como la que usamos en las clases, pero de este tema y con ella harás un esquema de lo que has de estudiar, para lo cual puedes usar el libro como apoyo. Igualmente añadirás las definiciones y ejemplos. Este trabajo lo debes hacer a mano en tu cuaderno.

3.- $\quad$ En tercer lugar, utilizando una serie de páginas webs, como:

Las Galaxias: http://www.xtec.es/ rmolins1/univers/es/galaxias.htm

Las Galaxias (2): http://www.astromia.com/universo/galaxias.htm

Las Galaxias (3): http://www.galeon.com/casanchi/ast/galaxi2.htm

Guía de Galaxias (documentos de Intranet)

Las galaxias se clasifican en: http://usuarios.lycos.es/ochacon/galaxias.htm

Galaxias del Grupo Local:http://www.galeon.com/casanchi/ast/galaxi1.htm 
Si hubiera algún problema con la conexión, deberá utilizar el documento de la página web interna del centro que el profesor le proponga.

Con ellas realizará varias actividades y con todo completará el esquema, los apuntes y lo ampliará.

4.- Después leerá en el libro la lección y la subrayará, completando el esquema anterior.

5.- Seguidamente realizaremos una o varias prácticas con los ordenadores de identificación de la velocidad de alejamiento de varias galaxias, así como de su clasificación. En la que rellenarás las hojas de prácticas que se te soliciten.

6.- Finalmente se realizará un test de los aprendizajes, destrezas y conocimientos adquiridos, y al terminar varios temas se hará un examen.

\section{Galaxy Formation: vídeos visibles desde Internet}

QuickTime (454K): http://archive.ncsa.uiuc.edu/Cyberia/Cosmos/Movies/ TheWorks1.mov

MPEG (238K): http://archive.ncsa.uiuc.edu/Cyberia/Cosmos/Movies/ TheWorks1.mpg [consulta 2006, 20 de junio 2006]

\section{REFERENCIAS BIBLIOGRAFICAS}

Adell, J. (2002). Internet en el aula: la caza del tesoro. Centre d'Educació i Noves Tecnologies. Universitat Jaume I.

Alonso y Gallego. (1987). Aprendizaje y Ordenador, Madrid. UNED.

Alonso Tapia, J. (1997). Motivar para el aprendizaje. Teoría y estrategias. Barcelona: Edebé.

Beltrán, J. A. (2003, a). Enseñar a Aprender. Universidad Complutense de Madrid. En el Congreso sobre la Novedad Pedagógica de Internet. Madrid: Educared.
Beltrán, J. A. (2003, b). Las TIC: Mitos, promesas y realidades. En el Congreso sobre la Novedad Pedagógica de Internet. Madrid. Educared.

Beltrán, J. A. (1996). Estrategias de aprendizaje. E J. A. Beltrán y C. Genovard: Psicología de la Instrucción. Madrid: Síntesis

Beltrán, J. A. (1983). Procesos, estrategias y técnicas. Madrid. Ed. Síntesis.

Beltrán, J. A. y Carbonell, J. L. (1999). Informe de Evaluación. Madrid: Consejería de Educación. Comunidad de Madrid. 
JOSÉ TORTAJADA

LAS WEBQUEST Y EL MODELO CAIT COMO MODELOS DE ENSEÑANZA APRENDIZAJE EN LA RED INTERNET

Beltrán, J. A. y Pérez, L. F. (2003). Cómo aprender con tecnología. En J. M.

Patino, J. A. Beltrán y L. F. Pérez: Cómo aprender con Internet. Madrid: Foro Pedagógico de Internet.

Beltrán, J.A. (1993). Aprender. En Procesos, estrategias y técnicas de aprendizaje. Madrid: Editorial Síntesis, S.A., pp. 1547.

Beltrán, J. A. (1993). Las estrategias. En Procesos, estrategias y técnicas de aprendizaje. Madrid: Editorial Síntesis, S.A., pp. 48-61.

Beltrán, J.A. (1993). Estrategias de repetición. Procedimientos nemotécnicos. En Procesos, estrategias y técnicas de aprendizaje. Madrid: Editorial Síntesis, S.A., pp. 253-262 y pp. 215-226.

Beltrán, J.A. (1991). Proceso de adquisición: Estrategias de elaboración. En Procesos, estrategias y técnicas de aprendizaje. Madrid: Editorial Síntesis, S.A., pp. 187214, 248-251,127-129.

Beltrán, J. A. (1993). Proceso de adquisición: Estrategias de selección y organización. En Procesos, estrategias y técnicas de aprendizaje. Madrid: Editorial Síntesis, S.A., pp. 122-126, 141-146.

Beltrán, J.A. (1995). Estrategias de aprendizaje. En J.A. Beltrán y J. Bueno (Eds.), Psicología de la educación. México: Alfaomega, pp. 307-331.

Bernie Dodge. (1995). WebQuests: A Technique for Internet-Based Learning . DISTANCE EDUCATOR 1: 10-13.

Blanco Suárez, S. et al. (2003). IES Ribera de Castilla, Valladolid. Disponible en: $\quad$ http://nogal.mentor.mec.es/ lbagoooo/html/educar 1.HTM [consulta 2006, 20 de junio 2006]
Echeverría, Javier (2001). Las TIC en educación. Revista Iberoamericana, 24

Jonassen, D. H. (2000). Computers as mindtools for schools. New Jersey: Prentice: Hall

Jonassen, D. H. Y Reeves , T. C. (1996). Learning with technology. En D. H.

Jonassen: Handbook of research for educational communications and technology, 693-719

Jonassen, D. H. (2000). Computers as mindtools for schools. New Jersey: Prentice: Hall

March, T. (1995). What's on the Web? $\mathrm{N}^{\circ}$ Julio - Agosto. 1995. "Computer-Using Educators" Newsletter.

Marqués Graells, P. (2000) Funciones y limitaciones de las tic en educación. Departamento de Pedagogía Aplicada, Facultad de Educación, UAB. Disponible en: http://dewey.uab.es/pmarques/ siyedu.htm [consulta 2006, 20 de junio 2006]

Marqués Graells, P. (2001). En Ideas para aprovechar el ciberespacio en educación Disponible en: http://dewey.uab.es/ pmarques/buenidea.htm

Martín Patino, Beltrán y Pérez. (2003). Cómo aprender con Internet, Foro Pedagógico de Internet, Madrid. Educared.

Martínez, R. (2003). Disponible en: http:// www.terra.es/personal/raumar/home. htm. [consulta 2006, 20 de junio 2006]

Paredes, A. Y Vásquez, C. (2003). Métodos y Técnicas de Estudio. Webs: Disponible en: http://sardis.upeu.edu.pe/ alfpa/ apoyo.htm 
Paredes Aguirre, A.. (2002) Disponible en: http://sardis.upeu.edu.pe/ alfpa/ apoyo.htm [consulta 2006, 20 de junio 2006]

Rosado L., y Tortajada, J. (2002, a). Uso de los ordenadores en la enseñanza de la geología planetaria usando Internet e Intranet, aplicados a Marte. Tecnologías de la Información y la Comunicación para el aprendizaje. Ed. Catalina M. Alonso y D. Gallego, Madrid. UNED.

Rosado L., y Tortajada, J. (2002, b). Enseñanza de la Astronomía con Internet y otras Nuevas Tecnologías. Didáctica de la Física y sus nuevas tendencias. (Manual 2003). Madrid. Ed. UNED.

Rosado L., y Tortajada, J. (2003). Formar al profesorado en la utilización de las Nuevas Tecnologías en la Enseñanza de las Ciencias. Didáctica de la Física y sus nuevas tendencias. (Manual 2003). Madrid. Ed. UNED.

Sestayo Fdez., Ma J. (2001). Formación de personal docente en NTs. A Coruña.

Starr, Linda. (2000). An Education World e-Interview With Bernie Dodge: Meet Bernie Dodge -- the Frank Lloyd Wright of Learning Environments! Education World (2000). Disponible en: http:// www.educationworld.com/a tech/ tech020.shtml, [Web Page, 05-2000] [2001, 06-06]. [consulta 2006, 20 de junio 2006]
Soler Areta, José Ma . (2003). Modelo CAIT: Estrategias para una nueva pedagogía. II Congreso Internacional de Educared.

Tortajada Perrote, J. (2001). Investigación planetaria con Internet. Ponencia. En el CD "La novedad pedagógica de Internet. I Congreso de Educared. Madrid. Ed. Ministerio de Educación.

Tortajada Perrote, J.(2001). Uso de Interneten la enseñanza de la Geología Planetaria. Madrid. CEP de Alcalá de Henares.

Tortajada Perrote, J. (2001). Uso de Internet en la enseñanza de la Geología Planetaria En comunicaciones del Congreso de Internet en la Educación, y la Educación en Internet. Madrid. Ed. Comunidad de Madrid.

Tortajada, J. (2002). "La novedad pedagógica de Internet. I Congreso de Educared. Ponencia: Investigación planetaria con Internet. Disponible en: $\quad$ http://www.educared.net/pdf/ congreso-i/Cx17invesplanetaria.PDF) [consulta 2006, 20 de junio 2006]

Tortajada, J. (2002)."La novedad pedagógica de Internet. I Congreso de Educared. Ponencia: Investigación de la contaminación local y mundial con Internet. Disponible en: www.educared. net/pdf/congreso-i/c8.pdf. [consulta 2006, 20 de junio 2006]

\section{PALABRAS CLAVE}

Aprendizaje, Astronomía, Biología, CAIT, Geología, Internet, Webquest.

\section{KEY WORDS}

Learning, Astronomy, Biology, CAIT, Geology, Internet, Webquest. 
JOSÉ TORTAJADA

LAS WEBQUEST Y EL MODELO CAIT COMO MODELOS DE ENSEÑANZA APRENDIZAJE EN LA RED INTERNET

\section{PERFIL ACADÉMICO DEL AUTOR}

José Tortajada Perrote, Biólogo por la Universidad Complutense de Madrid, Master en Psicología de la Educación por la Universidad Autónoma de Madrid, es Profesor de Enseñanza Secundaria con la categoría de Catedrático numerario de Bachillerato y actualmente es doctorando en la Facultad de Educación de la Universidad Nacional de Educación a Distancia (España). Ha realizado numerosas investigaciones, cursos y publicaciones sobre la actividad educativa, en especial utilizando las TIC.

Dirección postal:

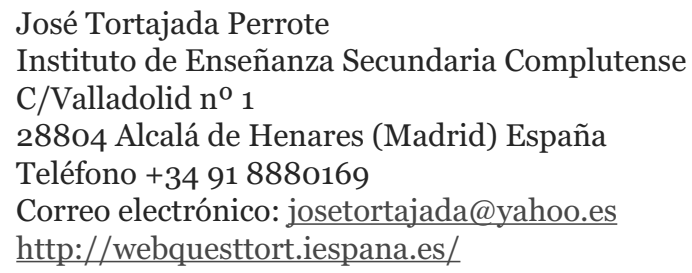

Fecha de entrega: 23.10.06

Fecha de aceptación: 20.12.06 